\title{
Microwave exfoliated graphene-based materials for flexible solid-state supercapacitor
}

\begin{abstract}
Herein, we report the simultaneous exfoliation and reduction of graphene oxide (GO) and graphene nanoplatelets (GNPs) by rapid microwave irradiation, to overcome the hurdles of their low electrical conductivity and tendency to restack, and realize their full potential as supercapacitor electrode materials. After microwave treatment, the $I_{D} / I_{G}$ value of the microwaved GO (MW-GO) increased by 0.11 , whereas the $I_{2 D} / I_{G}$ value of the microwaved GNPs (MW-GNPs) decreased by 0.48 , revealing that the graphene-based materials were reduced and exfoliated as observed in the Raman spectra. Morphological studies revealed a porous structure of MW-GO and loose stacked layers of MW-GNPs, which showed the exfoliation of the graphene-based materials. A supercapacitor device was constructed using a mixture of MW-GO, MW-GNPs, and polypyrrole and yielded a specific capacitance value of 137.2 $\mathrm{F} \mathrm{g}^{-1}$ with a cycling stability of $89.8 \%$ after 1000 charge/discharge cycles. The electrochemical performance of the device remains unchanged when bent continuously at $180^{\circ}$ because the cyclic voltammetry and galvanostatic charge/discharge curves remained the same after 50 bending repetitions. Therefore, the simultaneous reduction and exfoliation of these graphene-based materials by rapid microwaves provides a promising route for the scalable and cost-effective preparation of supercapacitor electrode materials.
\end{abstract}

Keyword: Flexible supercapacitor; Microwave irradiation; Graphene oxide; Graphene nanoplatelets; Graphene mixture; Graphene/polypyrrole mixture 